\title{
Um Levantamento dos Determinantes de Inovação em Softwares Educacionais
}

\author{
Rafael Santos Barbosa ${ }^{1}$, Ricardo André Cavalcante de Souza ${ }^{1}$ \\ ${ }^{1}$ Departamento de Computação (DC) \\ Universidade Federal Rural de Pernambuco (UFRPE) \\ Rua Dom Manoel de Medeiros, s/n, Dois Irmãos, CEP 52171-900, Recife-PE - Brasil \\ s.rafaelbarbosalgmail.com, ricardo.souzalufrpe.br
}

\begin{abstract}
Several studies report that innovative teaching-learning processes are usually aided by technology, especially using software. The identification of innovation drivers for software-supported education can be used to better guide the development, evaluation and selection of software for this purpose. This work then presents the result of a literature systematic mapping considering studies related to innovation factors in software aimed at education. From the set of 146 studies analyzed, 17 of these were selected because they were more relevant to the researched topic.
\end{abstract}

Resumo. Diversos estudos relatam que processos de ensino-aprendizagem inovadores são geralmente auxiliados por tecnologia, em especial pelo uso de softwares. A identificação dos determinantes de inovação para a educação apoiada por software pode ser usada para melhor orientar o desenvolvimento, avaliação e seleção de softwares para este propósito. Este trabalho apresenta o resultado de um mapeamento sistemático da literatura considerando estudos relacionados a fatores de inovação em softwares voltados para a educação. A partir do conjunto de 146 estudos analisados, 17 destes foram selecionados por serem mais relevantes ao tema pesquisado.

\section{Introdução}

Inovação refere-se a novas aplicações de conhecimentos, ideias, métodos e habilidades que podem gerar capacidades únicas e alavancar a competitividade de uma organização [Andersson et al., 2008]. Uma inovação é a implementação de um produto (bens e serviços) ou processo, novo ou significativamente melhorado [Mortensen, 2005].

Inovação é considerada um elemento-chave na economia baseada em conhecimento e fundamental para a melhoria contínua da educação e para o aumento dos resultados de aprendizagem, equidade, eficiência de custos e satisfação do estudante [Looney, 2009]. A inovação na educação se dá na melhoria das práticas pedagógicas e do processo de ensino e aprendizagem e desenvolvimento do estudante.

Educadores e instituições precisam estar abertos a mudanças, repensem as suas práticas, possibilitando o uso de novas propostas interdisciplinares, metodologias interativas e colaborativas, e permitindo que aconteça uma exploração de novos espaços que vão além dos limites da sala de aula [Kenski 2015]. 
VII Congresso Brasileiro de Informática na Educação (CBIE 2018)

Anais do XXIX Simpósio Brasileiro de Informática na Educação (SBIE 2018)

Segundo a OECD (2014), a inovação manifesta-se em diversas dimensões da educação, na forma de produtos ou processos. Um produto designa qualquer coisa que pode ser ofertada para um mercado visando atenção, aquisição, uso ou consumo e que pode satisfazer um desejo ou necessidade [Kotler, 2010]. Um processo é uma série de ações que produz alguma coisa ou que leva a um resultado em particular [MerriamWebster, 2016].

No contexto do processo educacional, a inovação está em estimular nos estudantes o desejo de aprender e em desenvolver as habilidades para "aprender a aprender" (por exemplo, capacidade para resolver problemas e análise crítica, aumentar a autonomia, iniciativa, flexibilidade, e assim por diante). Estudantes em ambientes onde o aprendizado visa desenvolver análise e pensamento crítico demonstraram retenção do conteúdo em longo prazo e melhor capacidade em aplicar o conhecimento no dia-a-dia [Looney, 2009].

Os produtos de software podem fornecer feedback valoroso sobre o aprendizado dos estudantes, mensurar processos de raciocínio e outras habilidades cognitivas complexas, e até mesmo examinar como os estudantes vão pensar sobre o problema [Looney, 2009]. Por exemplo, estudantes podem: (1) usar funções multimídia para mostrar como eles irão realizar um experimento físico ou alguma outra tarefa de resolução de problemas; (2) predizer, observar e explicar conceitos específicos; (3) desenvolver mapas conceituais para mostrar seus entendimentos dos processos e tais mapas podem então ser comparados com mapas de especialista [Bennet, 2001]; e (4) obter feedback do trabalho em tempo real.

Este trabalho visa apresentar o resultado de um mapeamento sistemático dos estudos mais recentes relacionados a determinantes e indicadores de inovação em softwares educacionais visando fornecer subsídios para o desenvolvimento, avaliação e seleção de softwares cujo propósito é apoiar o processo de ensino-aprendizagem.

Além desta introdução, este trabalho está organizado em mais três seções. A Seção 2 apresenta o Mapeamento Sistemático da literatura e a Seção 3 são apresentadas as considerações finais.

\section{Mapeamento Sistemático da Literatura}

O mapeamento sistemático da literatura, baseado no trabalho de Petersen et al. (2008), consiste de quatro etapas: Definição do escopo do mapeamento; Busca por estudos primários; Extração de dados; e Análise e síntese. A seguir são apresentadas a execução das etapas do mapeamento sistemático no contexto deste trabalho.

\subsection{Definição do Escopo do Mapeamento}

Nesta etapa foi realizado o planejamento do mapeamento sistemático, através de um protocolo (Tabela 1) com a estratégia para a busca e com uma questão de pesquisa (RQ do inglês Research Question) para orientar o processo. 
VII Congresso Brasileiro de Informática na Educação (CBIE 2018)

Anais do XXIX Simpósio Brasileiro de Informática na Educação (SBIE 2018)

Tabela 1. Fragmento do Protocolo do Mapeamento Sistemático

\begin{tabular}{|c|l|}
\hline \multirow{4}{*}{ Bases de pesquisa: } & $\begin{array}{l}\text { 1. IEEE Computer Society Digital Library } \\
\text { 2. Scielo }\end{array}$ \\
& $\begin{array}{r}\text { 3. ACM } \\
\text { 4. Scopus }\end{array}$ \\
& $\begin{array}{r}\text { 5. Google Scholar } \\
\text { 6. Springer }\end{array}$ \\
\hline Anos de Publicação & 2013 a 2017 \\
\hline \multirow{2}{*}{ Equipe Executora } & $\begin{array}{l}\text { (1) Estudante de mestrado em informática aplicada e (2) } \\
\text { orientador }\end{array}$ \\
\hline \multirow{2}{*}{ Questão de Pesquisa } & $\begin{array}{l}\text { Quais os determinantes e indicadores de inovação em } \\
\text { softwares educacionais? }\end{array}$ \\
\hline
\end{tabular}

\subsection{Busca por Estudos Primários}

Nesta etapa foi realizada a busca dos Estudos Primários nas bases de pesquisa. A busca de literatura a fim de verificar a natureza, extensão e a avaliação qualitativa dos trabalhos nas áreas de interesse resultam na identificação de trabalhos acadêmicos denominados Estudos Primários (EP). O maior desafio nesta etapa foi a construção da string de busca mais apropriada para o mapeamento sistemático. A construção da string de busca, baseada na proposta de Kitchenham (2006), consistiu na identificação de palavras-chave, a partir da RQ, e respectivos sinônimos, combinadas pelos conectores lógicos OR e AND. A primeira string de busca base resultante foi:

("education software assessment" OR "educational software" OR "educationals softwares" OR "educative software" OR "educatives softwares" OR "learning objects") AND ("measure innovation" OR "innovation indicators" OR "innovative indicators" OR "innovation drivers")

Para os resultados em português, foi utilizada a seguinte string de busca:

("avaliação de software educativo" OR "software educacional" OR "software educativo" OR "objetos de aprendizagem") AND ("medir inovação" OR "indicadores de inovação" OR "indicadores inovativos”)

Com a aplicação da string de busca nas bases de pesquisa, foram retornados 146 estudos primários, os quais foram catalogados e organizados por meio da ferramenta de software Mendeley ${ }^{1}$, conforme apresentado na Figura 1. Mendeley é uma ferramenta de software gratuita, usada como gerenciador e repositório de trabalhos científicos, com a possibilidade de compartilhar, fazer anotações de comentários e editar dados dos artigos [Zaugg, H, 2011]. Além disso, existe ainda a possibilidade da integração dos motores de busca com o Mendeley, facilitando a pesquisa, organização e avaliação dos artigos pesquisados.

\footnotetext{
${ }^{1}$ www.mendeley.com
} 
VII Congresso Brasileiro de Informática na Educação (CBIE 2018)

Anais do XXIX Simpósio Brasileiro de Informática na Educação (SBIE 2018)

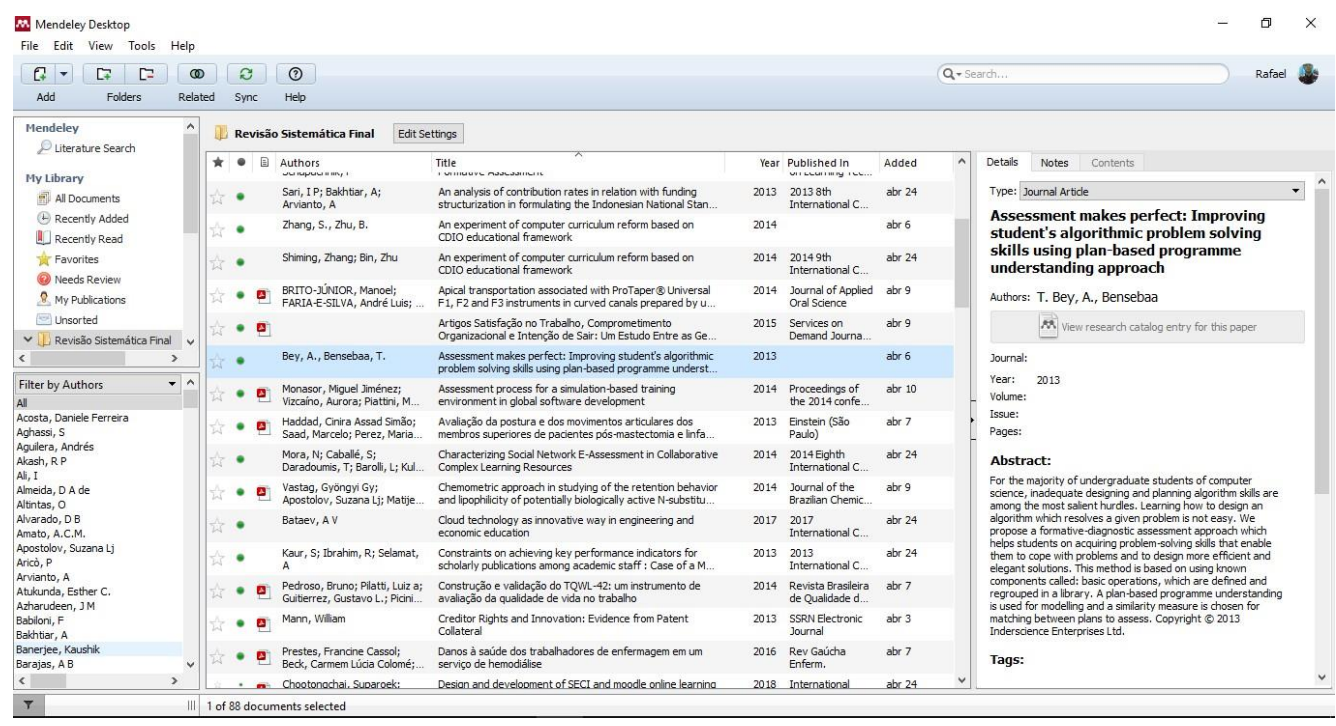

Figura 1. Organização dos Estudos Primários no Mendeley

\subsection{Extração de Dados}

A Extração de Dados consistiu na aplicação de um filtro, através de critérios de inclusão e exclusão, para identificação dos EP (considerados na etapa anterior) mais alinhados ao propósito do mapeamento sistemático. Os critérios de inclusão e exclusão servem para selecionar os estudos mais relevantes que respondam à questão de pesquisa [Arksey e O’Malley,2005; Petersen 2007], estes critérios estão apresentados na Tabela 2.

Foram definidos os seguintes Critérios de Inclusão: (1) artigos completos que identifiquem indicadores e determinantes de inovação em softwares educacionais; (2) artigos completos que tratam de avaliação de softwares educacionais e (3) artigos completos escritos em português e inglês. Para os Critérios de Exclusão, foram estabelecidos: (1) artigos publicados antes de 2013; (2) artigos com escopo não alinhado à RQ; (3) artigos repetidos e (4) trabalhos que não são artigos completos, tais como, resumos, palestras, apresentações, entre outros.

Inicialmente, foram realizadas leitura e análise dos títulos e resumos dos EP, selecionados na etapa anterior, e então aplicados os critérios de inclusão e exclusão definidos. Os artigos selecionados nesta etapa foram adicionados em uma pasta específica dentro do Mendeley. A Tabela 3 apresenta o quantitativo dos artigos selecionados nesta etapa, separados por ano de publicação (lado direito) e pela base de pesquisa (lado esquerdo).

Tabela 3. Quantidade de EP selecionados na Extração de Dados

\begin{tabular}{|c|c|c|c|c|}
\hline $\begin{array}{c}\text { Ano de } \\
\text { Publicação }\end{array}$ & $\begin{array}{c}\text { Quantidade de } \\
\text { Artigos }\end{array}$ & & $\begin{array}{c}\text { Base de } \\
\text { Pesquisa }\end{array}$ & $\begin{array}{c}\text { Quantidade de } \\
\text { Artigos }\end{array}$ \\
\hline 2013 & 7 & & Springer & 16 \\
\hline 2014 & 10 & IEEE & 15 \\
\hline 2015 & 4 & Scopus & 8 \\
\hline 2016 & 13 & ACM & 2 \\
\cline { 1 - 2 } & & & \\
\hline
\end{tabular}


VII Congresso Brasileiro de Informática na Educação (CBIE 2018)

Anais do XXIX Simpósio Brasileiro de Informática na Educação (SBIE 2018)

\begin{tabular}{|c|c|c|c|c|}
\hline 2017 & 8 & & Scielo & 0 \\
\cline { 1 - 1 } Total & \multirow{2}{*}{42} & Scholar & 1 \\
& & Total & 42 \\
\hline
\end{tabular}

\subsection{Análise e Síntese}

Nesta etapa foram definidos e aplicados critérios qualitativos (CQ) para análise qualitativa dos EP. Os resultados obtidos foram sintetizados por meios de gráficos e tabelas. Os seguintes critérios foram utilizados: CQ1: o estudo apresenta de forma clara um problema de pesquisa; CQ2: o estudo apresenta de forma apropriada os métodos usados para a coleta de dados; CQ3: o estudo responde ao respectivo problema de pesquisa; CQ4: o estudo demonstra alguma relação com determinantes e indicadores de inovação; CQ5: o estudo apresenta avaliação de softwares educacionais ou metodologias de ensino-aprendizagem inovadoras.

Para cada EP selecionado na etapa anterior, foi realizada leitura completa e realizada análise com base nos CQ. Para cada CQ analisado, foi definida a situação de AT (Atende), NT (Não Atende) ou NAP (Não se Aplica). A situação NAP foi utilizada para os trabalhos sem possibilidade de acesso ao conteúdo completo. Ao final da análise, foi definido um índice de aceitação (IA) do EP, de zero para aqueles sem qualquer AT a cinco para aqueles com todos os CQ com valor AT. Os EP com IA igual ou maior a três foram considerados como artigos aceitos (AA), os demais foram considerados artigos rejeitados (AR). A Tabela 4 apresenta os EP analisados de acordo com os CQ, bem como os respectivos resultados ${ }^{2}$.

Tabela 4. Fragmento da Tabela dos EP analisados pelos critérios qualitativos

\begin{tabular}{|l|l|c|c|c|c|c|c|c|}
\hline ID & \multicolumn{1}{|c|}{ Referência } & CQ1 & CQ2 & CQ3 & CQ4 & CQ5 & IA & Resultado \\
\hline 01 & $\begin{array}{l}\text { (Román-Gonzáles, Pérez- Gonzáles \& } \\
\text { Jiménez- Fernandez, 2017) }\end{array}$ & AT & AT & AT & NT & AT & 4 & AA \\
\hline 02 & (Maslak \& Doroshkevych, 2016) & NT & AT & AT & NT & NT & 2 & AR \\
\hline 03 & (Hussein, Seham et al., 2016) & AT & AT & AT & NT & NT & 2 & AR \\
\hline 04 & (Celeita, David et al., 2016) & AT & AT & NT & NT & NT & 2 & AR \\
\hline 05 & (Shiming, Zhang \& Bin, Zhu, 2014) & AT & AT & NT & NT & AT & 3 & AA \\
\hline 06 & (Ledesma \& Secades, 2013) & NAP & NAP & NAP & NAP & NAP & 0 & AR \\
\hline 07 & (Kowalski et al., 2013) & NP & AT & AT & NT & NT & 2 & AR \\
\hline 08 & (Caird, Hallett \& Potter, 2013) & AT & AT & AT & AT & NT & 4 & AA \\
\hline 09 & (Costello, 2014) & AT & NT & AT & NT & NT & 2 & AR \\
\hline 10 & (Veado et al., 2016) & AT & AT & NT & NT & AT & 3 & AA \\
\hline
\end{tabular}

\footnotetext{
${ }^{2}$ As referências completas dos 42 artigos analisados podem ser consultadas em https://goo.gl/vVAo73
} 
VII Congresso Brasileiro de Informática na Educação (CBIE 2018)

Anais do XXIX Simpósio Brasileiro de Informática na Educação (SBIE 2018)

\begin{tabular}{|c|c|c|c|c|c|c|c|c|}
\hline 11 & (Graf, Oppl \& Eckmaier,, 2017) & AT & NT & AT & NT & NT & 2 & AR \\
\hline 12 & (Benotti, Martinez \& Schapachni, 2018) & AT & AT & NT & NT & NT & 2 & AR \\
\hline 13 & (Renzella \& Cain, 2017) & AT & AT & NT & NT & AT & 3 & AA \\
\hline 14 & (Gama et al., 2016) & NT & AT & AT & NT & NT & 2 & AR \\
\hline 15 & (Navas et al., 2016) & AT & AT & NT & NT & NT & 2 & AR \\
\hline 16 & (Pantazos \& Vatrapu, 2016) & NT & AT & AT & NT & NT & 2 & AR \\
\hline 17 & (Lu et al, 2016) & NT & AT & NT & NT & AT & 2 & AR \\
\hline 18 & (Dos Santos Figueira et al., 2015) & AT & AT & NT & NT & AT & 3 & AA \\
\hline 19 & (Sichel, Woolf \& Floryan, 2014) & NT & AT & NT & NT & AT & 2 & AR \\
\hline 20 & (Trujillo-Aguilera et al., 2014) & NT & AT & NT & NT & NT & 1 & AR \\
\hline 21 & (Mora et al., 2014) & AT & AT & AT & AT & AT & 5 & AA \\
\hline 22 & (Luo et al., 2014) & NT & AT & NT & NT & NT & 1 & AR \\
\hline 23 & (Chen et al., 2014) & AT & NT & NT & NT & AT & 2 & AR \\
\hline 24 & (Ch \& Goteti, 2013) & AT & AT & NT & NT & AT & 3 & AA \\
\hline 25 & (Qidwai et al., 2013) & AT & AT & NT & NT & AT & 3 & AA \\
\hline 26 & (Makkonen et al., 2013) & AT & AT & NT & AT & NT & 3 & AA \\
\hline 27 & (Booyens et al., 2013) & AT & NT & AT & NT & NT & 2 & AR \\
\hline 28 & (Novak et al., 2014) & AT & AT & AT & AT & AT & 5 & AA \\
\hline 29 & (Tödtling \& Grillitsch, 2014) & AT & AT & NT & AT & AT & 3 & AA \\
\hline 30 & (Hueske et al., 2015) & NT & NT & AT & AT & NT & 2 & AR \\
\hline 31 & (Mihola et al., 2015) & NT & NT & AT & AT & NT & 2 & AR \\
\hline 32 & (Chor et al., 2015) & AT & AT & AT & AT & NT & 4 & AA \\
\hline 33 & (Jackson et al., 2016) & AT & NT & NT & AT & NT & 2 & AR \\
\hline 34 & (Rodrigez et al., 2016) & AT & NT & NT & AT & NT & 2 & AR \\
\hline 35 & (Mnif et al.,2016) & NT & NT & AT & AT & NT & 2 & AR \\
\hline 36 & (Ponchek, 2016) & AT & AT & NT & AT & NT & 3 & AA \\
\hline 37 & (Munir et al., 2016) & AT & AT & NT & AT & NT & 3 & AA \\
\hline 38 & (Kaneva \& Untura, 2017) & NT & NT & NT & AT & NT & 1 & AR \\
\hline 39 & (Selznick et al., 2017) & AT & AT & AT & AT & AT & 5 & AA \\
\hline 40 & (Martínez-román, et al., 2017) & AT & AT & NT & AT & NT & 3 & AA \\
\hline
\end{tabular}


VII Congresso Brasileiro de Informática na Educação (CBIE 2018)

Anais do XXIX Simpósio Brasileiro de Informática na Educação (SBIE 2018)

\begin{tabular}{|l|l|l|l|l|l|l|l|l|}
\hline 41 & (Rosenzweig, 2017) & AT & NT & NT & AT & NT & 2 & AR \\
\hline 42 & (Czarnitzki et al., 2014) & AT & NT & NT & AT & NT & 2 & AR \\
\hline
\end{tabular}

Ao final da análise com base nos CQ, foram considerados 17 EP aceitos (AA). $\mathrm{Na}$ síntese, inicialmente foi elaborado um Mapa Mental (Figura 2) com os principais termos encontrados nos EP considerados aceitos. O Mapa Mental é uma ferramenta gráfica usada para simplificar e organizar visualmente dados obtidos na pesquisa de campo, em diferentes níveis de profundidade e abstração (Vianna et al., 2012).

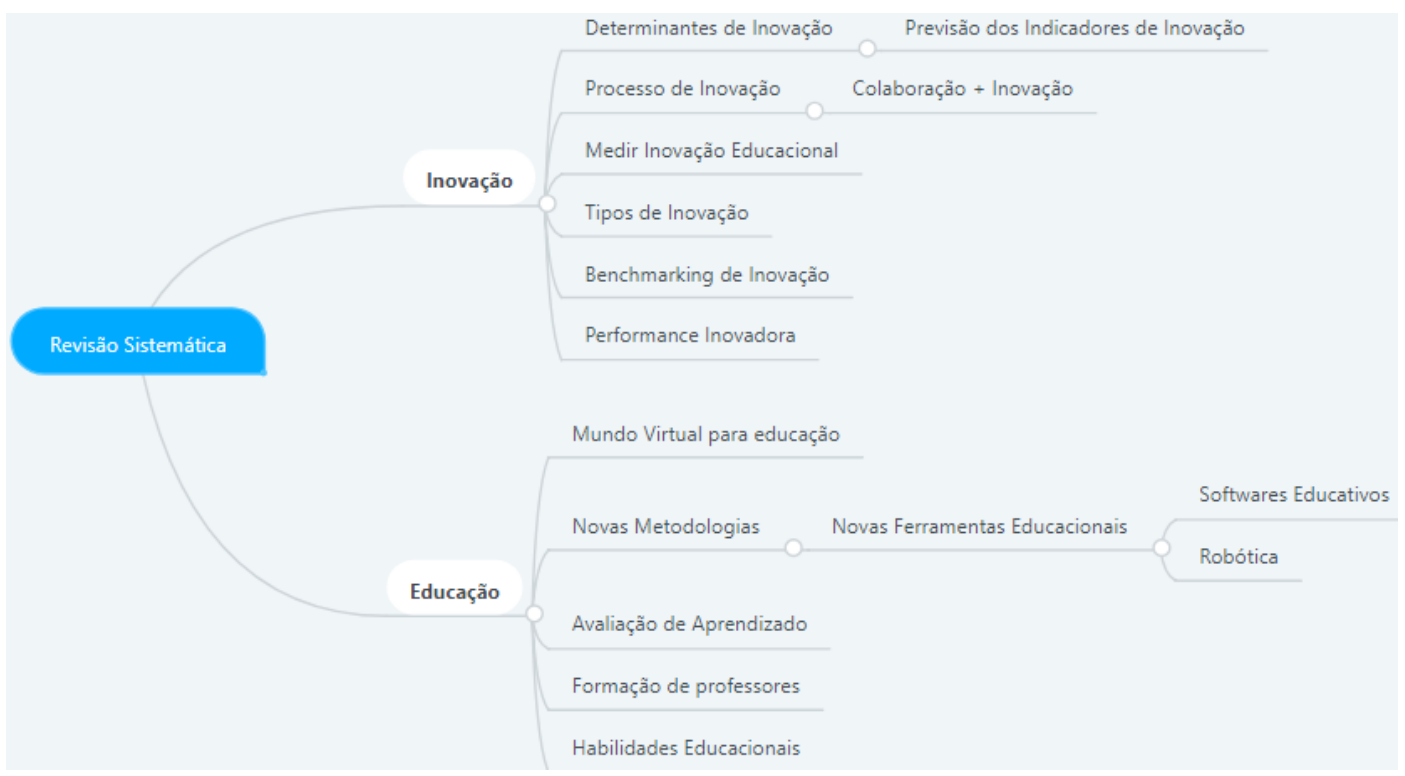

Figura 2. Mapa Mental dos Principais Termos Encontrados

Os resultados do mapeamento sistemático mostram: 02 artigos tratam sobre inovação educacional; 06 artigos versam sobre o desenvolvimento de novas metodologias com apoio de softwares educacionais; 04 artigos descrevem modelos de avaliação de inovação; 01 artigo especifica métricas de avaliação de software; 03 artigos tratam da avaliação de aprendizagem com suporte de softwares educacionais; e 05 artigos descrevem indicadores de inovação em geral. A Tabela 5 apresenta a temática abordada para os trabalhos considerados aceitos (AA).

Tabela 5. Síntese das Temáticas Abordadas

\begin{tabular}{|c|l|}
\hline Trabalhos (ID) & Temáticas Abordadas \\
\hline $\begin{array}{c}01 ; 05 ; 13 ; 18 ; \\
21 ; 24 ; 25\end{array}$ & $\begin{array}{l}\text { Novas metodologias educacionais e avaliação de aprendizagem com } \\
\text { suporte de software. }\end{array}$ \\
\hline 08 & $\begin{array}{l}\text { Modelo de avaliação de inovação a partir dos seguintes indicadores de } \\
\text { inovação: facilitadores, atividades organizacionais e resultados de } \\
\text { desempenho. }\end{array}$ \\
\hline 10 & $\begin{array}{l}\text { Proposição de ferramenta para avaliação de softwares educacionais } \\
\text { com base em métricas. }\end{array}$ \\
\hline
\end{tabular}


VII Congresso Brasileiro de Informática na Educação (CBIE 2018)

Anais do XXIX Simpósio Brasileiro de Informática na Educação (SBIE 2018)

\begin{tabular}{|c|c|}
\hline 26 & $\begin{array}{l}\text { Medição de inovação através dos seguintes indicadores: R\&D, patentes, } \\
\text { inovação (produto ou serviço), designs e marcas registradas, artigos } \\
\text { científicos, entre outros. }\end{array}$ \\
\hline 28 & $\begin{array}{l}\text { Processo de inovação centrado no usuário, baseado no uso de Avatares } \\
\text { em mundos virtuais e formação de comunidades de inovação. }\end{array}$ \\
\hline 29 & $\begin{array}{l}\text { Diagnóstico de inovação organizacional com base em critérios como: } \\
\text { inserção de produtos aprimorados; inovação de produtos para o } \\
\text { mercado; aplicação de novos processos ou estruturas organizacionais; } \\
\text { registro de patentes; desenvolvimento de novos produtos; e marketing. }\end{array}$ \\
\hline 32 & $\begin{array}{l}\text { Especificação de preditores de inovação: complexidade relativa; custo- } \\
\text { benefício e viabilidade; evidência e confiabilidade; facilitadores e } \\
\text { barreiras; experimentação; relevância e facilidade. }\end{array}$ \\
\hline 36 & Colaboração como mecanismo para melhorar o processo de inovação. \\
\hline 37 & $\begin{array}{l}\text { Mapeamento sistemático sobre processo inovação aberta em engenharia } \\
\text { de software. }\end{array}$ \\
\hline 39 & Inovação educacional e mensuração de inovação em sala de aula \\
\hline 40 & $\begin{array}{l}\text { Especificação de indicadores para atividades inovadoras: novos } \\
\text { produtos, aquisição de novos equipamentos, aquisição de novos } \\
\text { softwares, mudanças nos insumos, novos processos de produção, nova } \\
\text { organização interna, alteração na apresentação do produto. }\end{array}$ \\
\hline
\end{tabular}

De modo a responder à questão de pesquisa do mapeamento sistemático, conforme descrito no protocolo (Tabela 1), os indicadores e determinantes de inovação de softwares educacionais, resultantes da pesquisa na literatura, estão consolidados na Tabela 6 com a identificação das devidas fontes. Para determinar quais dos resultados encontrados foram determinantes (Det) ou indicadores (Ind) de inovação.

Tabela 6. Indicadores e Determinantes de Inovação

\begin{tabular}{|c|l|l|}
\hline $\begin{array}{l}\text { Indicador ou } \\
\text { Determinante }\end{array}$ & Descrição & $\begin{array}{l}\text { Fonte (ID do } \\
\text { EP) }\end{array}$ \\
\hline Det & Processo centrado no estudante & 28 \\
\hline Det & Formação de comunidades de inovação & 28 \\
\hline Det & Foco na motivação (funcional, experimental e social) & 28 \\
\hline Det & Viabilidade, confiabilidade e facilidade & 32 \\
\hline Det & Inovação organizacional & 29,26 \\
\hline Det & Pesquisa e desenvolvimento & 26 \\
\hline Ind & Registro de Patentes & 26,29 \\
\hline Ind & Artigos científicos e Certificações & 26 \\
\hline Ind & Alinhamento com normas e valores do usuário & 32 \\
\hline Ind & Inovação aberta & 37 \\
\hline Ind & Avaliação do desempenho & 08 \\
\hline
\end{tabular}

A partir da Tabela 6 podemos identificar alguns indicadores, que irão servir para mensurar a atividade inovadora do desenvolvimento de um software educacional. Os 
VII Congresso Brasileiro de Informática na Educação (CBIE 2018)

Anais do XXIX Simpósio Brasileiro de Informática na Educação (SBIE 2018)

indicadores vão permitir elaborar e avaliar o desempenho, resultados e os determinados fatores para inovação, esses aqui chamados de determinantes de inovação.

\section{Conclusão}

Este trabalho apresentou o resultado de um mapeamento sistemático que procurou responder a seguinte questão de pesquisa: "Quais os determinantes e indicadores de inovação em softwares educacionais?”. Os indicadores servem para mensurar inovação, já os determinantes servem como uma "ponte" até a inovação. A partir da execução do processo de mapeamento sistemático proposto por Petersen et al. (2008), foram analisados 146 estudos primários (artigos) e, destes, selecionados 17 considerados mais relevantes para a temática pesquisada. Os determinantes e indicadores de inovação levantados podem servir para auxiliar tomadas de decisão relacionadas a softwares de suporte ao processo educacional inovador.

Pelo mapeamento feito, foi identificado uma lacuna de que ainda não foi realizado nenhum trabalho que use os indicadores ou determinantes que tenham foco no desenvolvimento de software educacional. Desta forma, entre as oportunidades de pesquisa identificada está a elaboração de uma abordagem para avaliação de softwares educacionais com base em determinantes e indicadores de inovação.

\section{Referências}

Andersson, M.; Lndgren, R.; Henfridsoon (2008) O. Architectural knowledge in interorganizational IT Innovation. Journal of Strategic Information Systems, v. 1, n. 17, p. 19-38.

Arksey, H. e O’Malley, L. (2005). Scoping studies: towards a methodological framework. International Journal of Social Research Methodology, 8(1):19-32

Bennet, R. (2001) How the Internet will Help Large-Scale Assessment Reinvent Itself. Education Policy Analysis, v. 9, n. 5.

Cardoso, A. P. (2003) A receptividade à mudança e à inovação pedagógica: o professor e o contexto escolar. Perspectivas actuais/ Educação. Porto: Edições Asa.

Kenski, V. M. (2015) A urgência de propostas inovadoras para a formação de professores para todos os níveis de ensino. Rev. Diálogo Educ., v.15, n.45, p. 423441, maio/ago.

Kitchenham, B. (2004) Procedures for performing systematic reviews. Technical Report Technical Report TR/SE-0401, Keele University and NICTA.

Kitchenham, B. (2006). Empirical paradigm - the role of experiments. In Proceedings of the 2006 international conference on Empirical software engineering issues: critical assessment and future directions, pages 25-32, Berlin, Heidelberg. Springer-Verlag

Kotler, P.; Armstrong, G. (2010) Principles of Marketing. [S.1.]: Prentice Hall International.

Looney, J. W. (2009) Assessment and Innovation in Education. OECD Education Working Papers. [S.1.]: OECD Publishing. 
VII Congresso Brasileiro de Informática na Educação (CBIE 2018)

Anais do XXIX Simpósio Brasileiro de Informática na Educação (SBIE 2018)

Luna, A. J. D. O., Kruchten, P., Pedrosa, M. L. D. E., Neto, H. R., \& de Moura, H. P. (2014). State of the art of agile governance: a systematic review. arXiv preprint arXiv:1411.1922.

Magalhães, C. V., Santos, R. E., Da Silva, F. Q., \& Gomes, A. S. (2013). Caracterizando a pesquisa em informática na educação no Brasil: um mapeamento sistemático das publicações do SBIE. Simpósio Brasileiro de Informática na Educação-SBIE (Vol. 24, No. 1, p. 22).

Merriam-Webster. Merriam-Webster Dictionary. Disponivel em: https://www.merriamwebster.com/dictionary/process>. Acesso em: dez. 2016

Mortensen, P. S., \& Bloch, C. W. (2005). Oslo Manual-Guidelines for Collecting and Interpreting Innovation Data: Proposed Guidelines for Collecting and Interpreting Innovation Data. Organisation for Economic Cooporation and Development, OECD.

OECD (2014) Measuring Innovation in Education: A New Perspective, Educational Research and Innovation. [S.1.]: OECD Publishing.

Petersen, K.; Feldt, R.; Mujtaba, S.; Mattsson, M. (2008) Systematic Mapping Studies in Software Engineering. 12th International Conference on Evaluation and Assessment in Software Engineering (EASE). University of Bari, Italy.

Zaugg, H., West, R. E., Tateishi, I., \& Randall, D. L. (2011). Mendeley: Creating communities of scholarly inquiry through research collaboration. TechTrends, 55(1), $32-36$. 